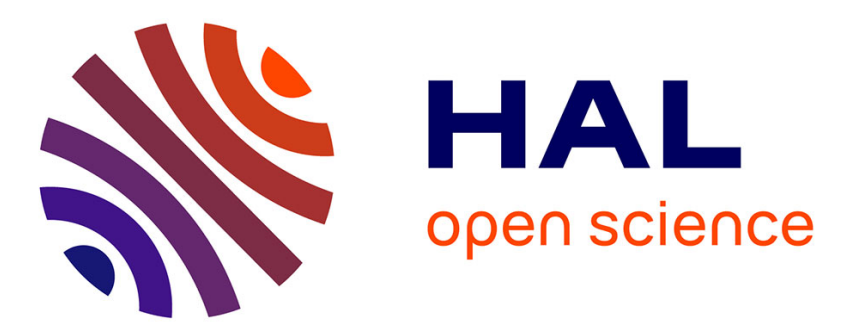

\title{
Simplified estimation of the flicker level induced by wave energy farms
}

\author{
Anne Blavette, Dara L O'Sullivan, Ray Alcorn, Michael G Egan, Tony W \\ Lewis
}

\section{- To cite this version:}

Anne Blavette, Dara L O’Sullivan, Ray Alcorn, Michael G Egan, Tony W Lewis. Simplified estimation of the flicker level induced by wave energy farms. IEEE Transactions on Sustainable Energy , 2016, 7 (3), 10.1109/TSTE.2016.2535327 . hal-01403251

\section{HAL Id: hal-01403251 \\ https://hal.science/hal-01403251}

Submitted on 25 Nov 2016

HAL is a multi-disciplinary open access archive for the deposit and dissemination of scientific research documents, whether they are published or not. The documents may come from teaching and research institutions in France or abroad, or from public or private research centers.
L'archive ouverte pluridisciplinaire HAL, est destinée au dépôt et à la diffusion de documents scientifiques de niveau recherche, publiés ou non, émanant des établissements d'enseignement et de recherche français ou étrangers, des laboratoires publics ou privés. 


\title{
Simplified estimation of the flicker level induced by wave energy farms
}

\author{
Anne Blavette, Dara L. O’Sullivan, Ray Alcorn, Michael G. Egan and Tony W. Lewis
}

\begin{abstract}
Wave energy farms may cause voltage flicker on the local grid to which they will be connected due to the strong fluctuations that their output power may present. IEC standard 61400-21 describes methods for estimating the flicker level for different short-circuit ratios as well as for different numbers of devices composing the farm. This method was initially developed for wind farms but is applicable to wave energy farms as well. However, besides the short-circuit ratio and the number of devices composing the farm, the grid impedance angle has also a strong influence on flicker. Despite this, no method exists in the literature for estimating flicker as a function of this variable. This paper presents the results of a study intended to fill this gap by focusing on developing a simplified method for estimating the flicker level induced by a wave energy farm as a function of the grid impedance angle. The results obtained through this method are compared with those obtained from numerical load flow simulations performed with PowerFactory. These simulations were based on experimental power output time series of a wave energy prototype deployed at sea as part of the European CORES project. The voltage profiles thus generated were then processed by means of a flickermeter compliant with IEC standard 61000-4-15.
\end{abstract}

Index Terms-Flicker, wave power, impedance angle

\section{INTRODUCTION}

$\mathbf{M}$ ANY maritime countries envisage to harness sea wave energy for producing electricity. However, the power fluctuations generated by wave devices, which are mostly oscillating bodies with little to no means of storage, may induce voltage fluctuations on the network. This voltage fluctuations may themselves create a phenomenon called flicker which is detailed in Section II. It must be emphasized that flicker must be maintained below a defined level in order to meet power quality requirements enforced by grid operators. Failing to meet these power quality requirements could lead to grid connection denial. Hence, flicker must be carefully evaluated from the early stages of the device design process.

Besides the wave device type and the wave farm design, three variables can strongly influence the flicker level induced by a wave energy farm: the short-circuit ratio at the point of connection (i.e. the ratio of the short-circuit power to the farm rated power) as well as its impedance angle $\Psi_{k}$, and the number of devices composing the farm. As it will be presented in the state of the art (Section II), the short-term

A. Blavette is with SATIE (CNRS unit 8029), Rennes, France, e-mail: anne.blavette@ens-rennes.fr

D. L. O'Sullivan is with Analog Devices, Cork, Ireland.

R. Alcorn and T. Lewis are with MaREI, University College Cork, Ireland.

M. G. Egan, now retired, was with the Electrical Engineering Department, University College Cork, Ireland.

Manuscript received December DD, 2015. flicker level $\left(P_{s t}\right)$ is inversely proportional to the short-circuit ratio. A flicker summation law can also be used to determined the flicker level generated by a wave energy farm based on the flicker level generated by a single device [1]. However, no sufficiently precise method exists for estimating the flicker level with respect to the impedance angle at the point of connection. Hence, the relation between the short-term flicker level $P_{s t}$ and the impedance angle $\Psi_{k}$ was studied and the results are shown in this paper.

The paper is divided as follows: Section II summarizes the state of the art on this research question, Section III describes the theoretical analysis performed as part of this study and Sections IV details the numerical models as well as the simulations carried out. The results are presented in Section V. The simplified method proposed for flicker level estimation and the conclusion are described in Section VI.

\section{STATE OF THE ART}

Flicker level is evaluated based on the perception of light intensity variations [2]. The lighting equipment considered in this standard is an incandescent light bulb. It must be noted that basing flicker level assessment on this type of bulb may represent a worst case scenario as a number of lighting equipment types such as LEDs or compact fluorescent lamps have a lower flicker response to low-frequency voltage modulation as generated by wave energy farms [3], [4]. Also, this standard is now considered as incomplete as it does not include flicker generation from beat frequencies between the fundamental and interharmonics [5], [6], [7]. However, in the absence of widely agreed guidelines or standards on the response of different types of lighting and electrical equipment, the recommendations established by IEC standard 61000-415 still remain the reference. In addition, the influence of beat frequencies between the fundamental and interharmonics is deemed negligible in the case considered here, as it will be explained later. Hence, the flicker evaluation procedure detailed in IEC standard 61000-4-15 was retained for the study presented in this paper.

IEC standard 61400-21 [1] constitutes the reference regarding the power quality assessment of wind turbines. The method described in this standard is based on an ideal network, i.e. presenting a constant system frequency and a constant voltage. This procedure has also been used as a guidance for assessing the grid impact of wave energy devices or farms in the absence of dedicated assessment protocols [8], [9]. The procedure described in IEC standard 61400-21 is composed of three stages. First, the instantaneous line current output by the 


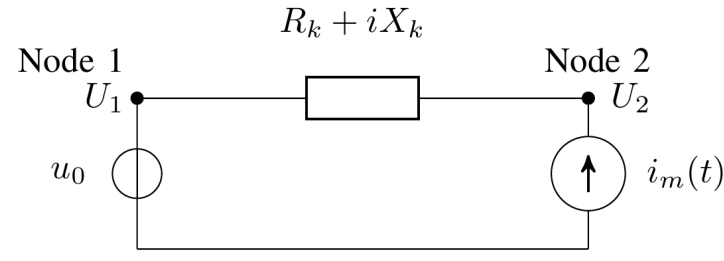

Fig. 1. Fictitious grid as described in IEC standard 61400-21

considered wind turbine is measured. The wind turbine should be connected directly to the medium or high voltage network (greater than $1 \mathrm{kV}$ ). The rest of the analysis is performed by means of numerical simulations based on a fictitious grid composed of a constant voltage source $u_{0}(t)$ in series with a resistance $R_{k}$, an inductance $L_{k}$ and a current source $i_{m}(t)$ representing the wind turbine, as shown in Fig.1. The current source outputs the instantaneous line current as measured at the wind turbine terminals during the first stage. Using this fictitious grid, in which the amplitude of the source $u_{0}(t)$ is constant, enables to perform tests in which the wind turbine is the sole source of voltage disturbance. Consequently, the results are independent of the grid conditions at the test site where the instantaneous line current has been measured. The impedance angle $\Psi_{k}$ and the short-circuit level $S_{k}$ of the fictitious grid can be expressed as:

$$
\tan \left(\Psi_{k}\right)=\frac{2 \pi f_{g} L_{k}}{R_{k}}=\frac{X_{k}}{R_{k}} \text { and } S_{k}=\frac{U_{n}^{2}}{\sqrt{R_{k}^{2}+X_{k}^{2}}}
$$

where $f_{g}$ is the grid frequency equal to $50 \mathrm{~Hz}$ or $60 \mathrm{~Hz}$ (depending on the geographical regions considered), and $U_{n}$ is the rms value of the grid nominal voltage. Then, the corresponding short-term flicker level $P_{s t}$ is computed by means of a flickermeter compliant with IEC standard 610004-15. IEC standard 61400-21 defines also a flicker coefficient $c\left(\Psi_{k}\right)$ which is dependent on the wind turbine considered and on the impedance angle $\Psi_{k}$ only, such as:

$$
P_{s t}=c\left(\Psi_{k}\right) \frac{S_{n}}{S_{k}}
$$

where $S_{n}$ is the rated apparent power of the wind farm. Hence, the flicker level corresponding to any short-circuit ratio can be directly determined based on the flicker coefficient $c\left(\Psi_{k}\right)$. In similar fashion, IEC standard 61400-21 defines a flicker summation law such as:

$$
P_{s t, t o t}=\sqrt{\sum_{i}^{N_{d}} P_{s t, i}^{2}}
$$

where $P_{s t, t o t}$ is the flicker level generated by a wave energy farm, $P_{s t, i}$ is the flicker level generated by device $i$, and $N_{d}$ is the number of devices composing the farm. Hence, power system simulations for a single value of the short-circuit ratio and for a single value of the device number only are necessary, as the flicker level corresponding to other conditions in terms of grid strength and device number can be directly derived based on (2) and (3).
However, IEC standard 61400-21 does not provide any formula from which the flicker level for a grid impedance $\Psi_{k}$ could be derived from a known flicker level corresponding to a grid impedance angle $\Psi_{k 0}$. It is important to recall that the influence of the grid impedance angle on flicker has been demonstrated to be potentially significant [9], [10]. Consequently, power system simulations have to be performed individually for each grid impedance angle $\Psi_{k}$ in order to determine the corresponding flicker level. In particular, IEC standard 61400-21 recommends to evaluate the flicker level for four different values of the impedance angle, namely $30^{\circ}$, $50^{\circ}, 70^{\circ}$ and $85^{\circ}$. In [11], an analytical formula for estimating the amplitude of the voltage variations (on which the flicker level is dependent) due to the injection of wind power as a function of the impedance angle has been considered. If the fictitious grid shown in Fig. 1 is considered, this formula can be written as:

$$
U_{2}=\sqrt{c-\sqrt{c^{2}-d}}
$$

where $c=U_{1}^{2} / 2-\left(R_{k} P+X_{k} Q\right), d=\left(P^{2}+Q^{2}\right)\left(R_{k}^{2}+X_{k}^{2}\right)$ and $P$ and $Q$ are the active and the reactive power output by the turbine. This simplified approach has been further developed in order to be used as a preliminary flicker level assessment study dedicated to wave energy [10]. The objective of this latter research work was to determine the minimum short-circuit ratio for which a wave energy device complies with the grid operator's requirements in terms of flicker. The active power output is assumed to be continuously oscillating at the most sensitive frequency (in terms of flicker) which the wave devices can generate (i.e. $0.4 \mathrm{~Hz}$ ). In addition, this power output profile is assumed to be of rectangular form. All these conditions constitute a worst-case scenario. Hence, the flicker level results obtained through this method are not supposed to be held as representative of the flicker level generated by a wave energy farm. However, they help to determine whether more detailed flicker level assessment studies need to be performed. Another work has focused on developing an intrinsic flicker severity index independent of both the short-circuit ratio and of the grid impedance angle [12]. However, this paper assumed that electrical lines are lossless, which can lead to a significant error level when sufficiently resistive networks, as it is the case in distribution networks, are considered. Also, estimating the intrinsic flicker severity index requires using power profiles generated by the wave energy farm for a wide range of sea-state conditions. However, obtaining such data necessitates developing a numerical model of the wave energy converter considered which already proved to represent a tremendous task in the wind energy industry [13]. In addition, these models are very likely to be provided as "black-boxes" which can make them very complex to debug and to use in conjunction with other such models developed in an uncoordinated manner. It is in this context that the ocean energy industry has turned towards an alternative modeling approach called "generic modeling" [14]. A generic model consists of a generic, publicly-available structure which is parameterisable by means of data which developers can provide. The research work presented in this paper has adopted a similar approach in order to define a method for estimating 
the flicker level generated by a wave energy farm for any impedance angle and which does not require commerciallysensitive data. Doing otherwise could indeed lead to the development of methods which would be inapplicable due to a lack of available data. This method is hoped to contribute in further reducing the number of power system simulations required for a flicker assessment study in similar fashion to (2) and (3). This method could also facilitate the comparison of the results obtained from different flicker assessment case studies. So far, these studies are usually performed based on different values of the grid impedance angle [18], [19], [20]. Also, data on the power profiles output by a wave energy is usually not publicly available. Hence, in the absence of a method linking the flicker level to the grid impedance angle, comparing the flicker level generated by two different farms (for instance based on two different wave device technologies) at two different test sites is impossible.

\section{THEORETICAL ANALYSIS}

\section{A. Formula linking the flicker level to the impedance angle}

1) Introduction: The objective of Section III-A is to define a formula from which the flicker level $P_{s t}\left(\Psi_{k}\right)$ could be defined based on a known flicker level $P_{s t}\left(\Psi_{k_{0}}\right)$. It will be demonstrated that the ratio of the flicker level $P_{s t}$ (corresponding to a voltage profile $v(t)$ ) to the voltage difference $\Delta V$ of this profile (defined as the difference between the maximum and the minimum) is a constant. Any voltage profile $v(t)$ can be modeled as:

$$
v(t)=\left(V_{a}+V_{f}(t)\right) \sin \left(\omega_{g} t\right)
$$

where $V_{a}$ is the average voltage, $V_{f}(t)$ represents the voltage fluctuations around this value and $\omega_{g}$ is the radian grid frequency. The dependence of the average voltage $V_{a}$ and of the amplitude of the voltage fluctuations $V_{f}(t)$ to the impedance angle $\Psi_{k}$ is proposed to be modeled as:

$$
\left\{\begin{array}{l}
V_{a}\left(\Psi_{k}\right)=k_{1}\left(\Psi_{k}\right) V_{a}\left(\Psi_{k_{0}}\right) \\
V_{f}\left(t, \Psi_{k}\right)=k_{2}\left(\Psi_{k}\right) V_{f}\left(t, \Psi_{k_{0}}\right)
\end{array}\right.
$$

where $k_{1}\left(\Psi_{k}\right)$ and $k_{2}\left(\Psi_{k}\right)$ are scale factors corresponding to a given impedance angle $\Psi_{k}$. The terms $V_{a}\left(\Psi_{k_{0}}\right)$ and $V_{f}\left(t, \Psi_{k_{0}}\right)$ are respectively the average voltage and the voltage fluctuations corresponding to a given impedance angle $\Psi_{k_{0}}$. It is also assumed that the average voltage $V_{a}\left(\Psi_{k}\right)$ remains relatively close to unity, which was confirmed by the simulation results (see Section V). Hence the scale factor $k_{1}\left(\Psi_{k}\right)$ can be considered as almost constant as a function of the impedance angle and approximately equal to unity. So, (5) can be reformulated as:

$$
v\left(t, \Psi_{k}\right) \approx\left[V_{a}\left(\Psi_{k_{0}}\right)+k_{2}\left(\Psi_{k}\right) V_{f}\left(t, \Psi_{k_{0}}\right)\right] \sin \left(\omega_{g} t\right)
$$

For the sake of clarity, the scale factor $k_{2}\left(\Psi_{k}\right)$, the average voltage $V_{a}\left(\Psi_{k_{0}}\right)$, the voltage fluctuations $V_{f}\left(t, \Psi_{k_{0}}\right)$ and the voltage profile $v\left(t, \Psi_{k_{0}}\right)$ will be expressed as $k_{2}, V_{a_{0}}, V_{f_{0}}(t)$ and $v_{0}(t)$ respectively in the rest of the paper. Voltage fluctuations $V_{f_{0}}(t)$ can be expressed as a Fourier series whose period
$T$ is equal to the time during which the voltage profile $v(t)$ is observed. Hence, (7) can be rewritten as:

$$
v(t) \approx\left(V_{a_{0}}+k_{2} \sum_{n=-N}^{N} c_{n} e^{-\frac{2 i \pi n t}{T}}\right) \sin \left(\omega_{g} t\right)
$$

where $N \in \mathbb{N}, n \in \mathbb{N}^{*}$ and $c_{n}$ is the Fourier coefficient of rank $n$.

2) Flicker level $P_{s t}$ : A flickermeter is composed of five blocks. Block 1 of the flickermeter per-unitizes the incoming voltage signal $v(t)$. Block 2 consists of a squaring multiplier. The output signal $\mathrm{O}_{2}(t)$ of Block 2 is thus composed of several terms based on the product of terms oscillating at the grid frequency by terms oscillating at a frequency lying in a much lower range defined by the wave power fluctuations. More precisely, the frequency range of interest for wave energy conversion purposes goes from $0.05 \mathrm{~Hz}$ to $0.2 \mathrm{~Hz}$ [21]. Considering that a wave energy device generates power twice per wave cycle on average, the frequency range of its output power fluctuations spans between $0.1 \mathrm{~Hz}$ and $0.4 \mathrm{~Hz}$. This is also the frequency range of the voltage fluctuations which this device induces on the local grid. Hence, the product of terms oscillating at the grid frequency by terms oscillating at a frequency defined by wave power fluctuations is approximately equal to the product of the amplitudes of both types of terms times a term oscillating at the grid frequency. Hence, output signal $\mathrm{O}_{2}(t)$ of Block 2 can be approximated as:

$$
\begin{aligned}
O_{2}(t) & =v(t)^{2} \approx \frac{1}{2}\left(V_{a_{0}}^{2}+2 k_{2} V_{a_{0}} \sum_{n=-N}^{N} c_{n} e^{-\frac{2 i \pi n t}{T}}\right. \\
& +k_{2}^{2} \sum_{n=-N}^{N} \sum_{m=-N}^{N} c_{n} c_{m} e^{-\frac{2 i \pi(n+m) t}{T}} \\
& -V_{a_{0}}^{2} \cos \left(2 \omega_{g} t\right)-2 k_{2} V_{a_{0}} \sum_{n=-N}^{N} c_{n} e^{-2 i \omega_{g} t} \\
& \left.-k_{2}^{2} \sum_{n=-N}^{N} \sum_{m=-N}^{N} c_{n} c_{m} e^{-2 i \omega_{g} t}\right)
\end{aligned}
$$

where $m \in \mathbb{N}$. Block 3 consists of two filters in series. The first one is a pass-band filter whose cut-off frequencies are equal to $0.05 \mathrm{~Hz}$ and $35 \mathrm{~Hz}$. The second filter applies a gain $g_{n}$ to each sinusoidal term which depends on the frequency of this latter. In the frequency range within which these oscillating terms lie $([0.1 \mathrm{~Hz}, 0.4 \mathrm{~Hz}])$, the gain $g_{n}$ ranges between 0.01 and 0.04 . Hence, the output signal of Block 3 can be expressed as:

$$
\begin{aligned}
O_{3}(t) & \approx k_{2} V_{a_{0}} \sum_{n=-N}^{N} g_{n} c_{n} e^{-\frac{2 i \pi n t}{T}} \\
& +\frac{k_{2}^{2}}{2} \sum_{n=-N}^{N} \sum_{m=-N}^{N} g_{(n+m)} c_{n} c_{m} e^{-\frac{2 \pi(n+m) t}{T}}
\end{aligned}
$$

The term $V_{a_{0}}$ is of the order of magnitude of unity, as the average voltage is expected to remain within 1 per unit $\pm 10 \%$ maximum, as required in the most permissive grid codes. The terms $g_{n}$ and $g_{n+m}$ are both of the same order of magnitude, 
as indicated in the previous paragraph. Assuming that both $c_{n}, c_{m}<<1$, the amplitude $c_{n} c_{m}$ can be considered as much smaller than the amplitude $c_{n}$ (i.e. $c_{n} c_{m}<<c_{n}$ ). Hence, for a scale factor $k_{2}$ sufficiently small, signal $O_{3}(t)$ can be approximated by its first term only such as:

$$
O_{3}(t) \approx k_{2} V_{a_{0}} \sum_{n=-N}^{N} g_{n} c_{n} e^{-\frac{2 i \pi n t}{T}}
$$

Block 4 consists of a squaring multiplier, of a gain $K$ and of a first-order low-pass filter of time constant equal to $0.3 \mathrm{~s}$. All these sub-blocks are in series. The output signal $O_{4}(t)$ of Block 4 (also called the instantaneous flicker) is processed by a classifier in order to determine the amount of time during which it remains between two consecutive flicker levels $F_{h-1}$ and $F_{h}(h \in \mathbb{N})$. These levels range between 0 and the maximum instantaneous flicker level $F_{\max }$ :

$$
\begin{aligned}
F_{\max } & =\max \left(O_{4}(t)\right) \\
& =K k_{2}^{2} V_{a_{0}}^{2} \max \left(\left(\sum_{n=-N}^{N} g_{n} c_{n} e^{-\frac{2 i \pi n t}{T}}\right)^{2}\right)
\end{aligned}
$$

Based on the results of the classifier, a cumulative probability function (CPF) is generated in Block 5. The flicker level $P_{s t}$ is calculated based on the percentiles $P_{q}$ of the CPF. These percentiles represent the flicker level exceeded for $q \%$ of the time during 10 minutes. The percentiles $P_{q}$ are then used to calculate the flicker level $P_{s t}$, as it will be detailed later. These percentiles are proportional to the maximum instantaneous flicker level $F_{\max }$, which can be expressed as:

$$
P_{q} \propto F_{\max }=K k_{2}^{2} V_{a_{0}}^{2} \max \left(\left(\sum_{n=-N}^{N} g_{n} c_{n} e^{-\frac{2 i \pi n t}{T}}\right)^{2}\right)
$$

This can be demonstrated by means a simple example based on a sinusoidal input signal. However, this simple example is generalizable to more complex input signals. As shown in Fig. 2, in order to create the CPF, the instantaneous flicker level (i.e. the input signal $O_{4}(t)$ to the classifier) is divided into $N_{c}$ classes. The time which this signal spends in each class $h$ is computed. The number of classes $N_{c}$ being fixed, each flicker level $F_{h}=F_{\max } h / N_{c}$ corresponding to a class $h$ is proportional to the maximum instantaneous flicker level $F_{\max }$. A linear classification is shown in Fig. 2 for the sake of simplicity but the same rationale applies to a logarithmic distribution which is also widely used. Hence, if the input signal has the same "shape" (e.g. sinusoidal at a given frequency), then the $\mathrm{CPF}$ curve normalized by the maximum instantaneous flicker level $F_{\max }$ is independent of the amplitude $F_{\max }$ of this signal. This is illustrated in Fig. 3 which shows the normalized CPF curve for several sinusoidal input signals having different amplitudes $\Delta V / 2$. The existence of this normalized CPF curve implies the existence of normalized percentiles $p_{q}$ which are constant for a given voltage profile "shape", thus independent of its amplitude. In other words, these normalized percentiles $p_{q}$ are independent of the scale factor $k_{2}$, and dependent only on the reference voltage profile $v_{0}(t)$. Consequently, the

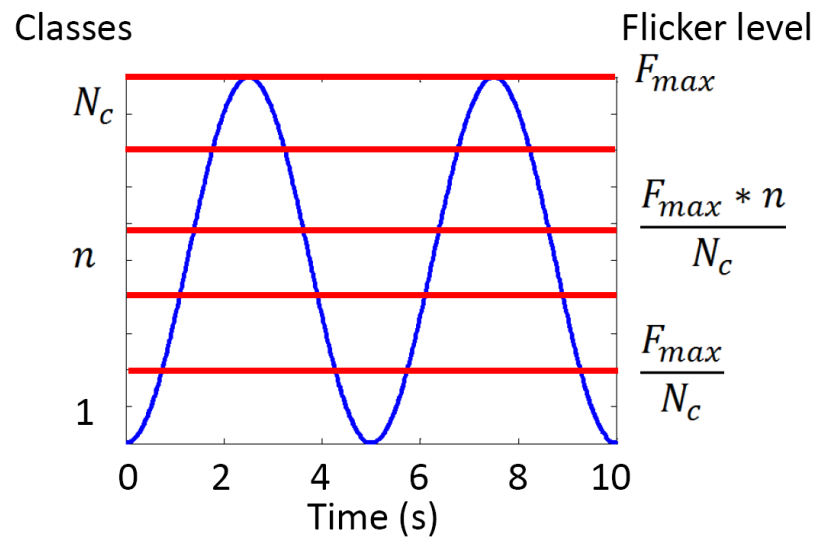

Fig. 2. Instantaneous flicker level as a function of time. The signal is divided into $N_{c}$ classes corresponding each to a flicker level $F_{\max } h / N_{c}$.

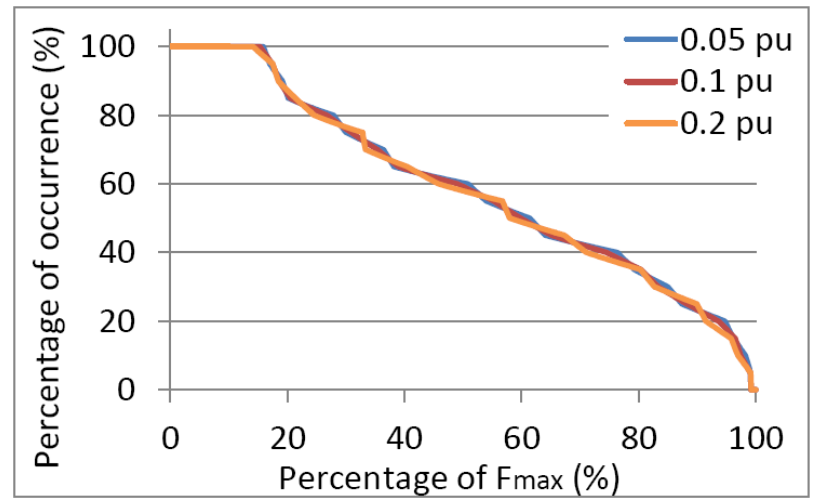

Fig. 3. Normalized cumulative probability function (CPF) for different values of the voltage difference $\Delta V(0.05 \mathrm{pu}, 0.1 \mathrm{pu}$ and $0.2 \mathrm{pu})$

expression of the absolute percentiles $P_{q}$ can be reformulated as:

$P_{q} \propto C k_{2}^{2} p_{q}$ with $C=K V_{a_{0}}^{2} \max \left(\left(\sum_{n=-N}^{N} g_{n} c_{n} e^{-\frac{2 i \pi n t}{T}}\right)^{2}\right)$

where $C$ depends on a given reference voltage profile $v_{0}(t)$ corresponding to a reference impedance angle $\Psi_{k_{0}}$. In other words, $C$ is constant for a given reference voltage profile and is therefore independent of the impedance angle $\Psi_{k}$.

Percentiles $P_{q}$ are used to calculate the flicker level $P_{s t}$ such as:

$$
P_{s t}=\sqrt{a_{1} P_{0.1}+a_{2} P_{1 s}+a_{3} P_{3 s}+a_{4} P_{10 s}+a_{5} P_{50 s}}
$$

where $a_{1}, a_{2}, a_{3}, a_{4}, a_{5}$ are constants. Percentiles $P_{q s}$ (where $q=[1 ; 3 ; 10 ; 50])$ are averaged values of percentiles $P_{q}$ based on $P_{q}$ itself and on the values of neighbouring percentiles. Equation (15) can be reformulated based on the normalized percentiles $p_{q}$ described in (14) as:

$$
\begin{aligned}
P_{s t} & =\sqrt{\left(C k_{2}^{2}\right)\left(a_{1} p_{0.1}+a_{2} p_{1 s}+a_{3} p_{3 s}+a_{4} p_{10 s}+a_{5} p_{50 s}\right)} \\
& =k_{2} P_{s t_{0}}
\end{aligned}
$$


where $P_{s t_{0}}$ is the flicker level corresponding to the voltage profile $v_{0}(t)$. The last equation can be reformulated in a more general manner as:

$$
P_{s t}\left(\Psi_{k}\right)=k_{2}\left(\Psi_{k}\right) P_{s t}\left(\Psi_{k_{0}}\right)
$$

3) Voltage difference $\Delta V$ : The voltage difference $\Delta V$ is defined as the difference between the maximum amplitude of the voltage profile $v(t)$ and its minimum. It can be expressed as:

$$
\begin{aligned}
\Delta V & =\max \left(V_{a}+V_{f}(t)\right)-\min \left(V_{a}+V_{f}(t)\right) \\
& =k_{2}\left[\max \left(V_{f_{0}}(t)\right)-\min \left(V_{f_{0}}(t)\right)\right]
\end{aligned}
$$

This can be reformulated in a more general manner as:

$$
\Delta V\left(\Psi_{k}\right)=k_{2}\left(\Psi_{k}\right) \Delta V\left(\Psi_{k_{0}}\right)
$$

4) Ratio $P_{s t} / \Delta V$ : Based on (17) and (19), the ratio of the flicker level $P_{s t}$ to the voltage difference $\Delta V$ can be expressed as:

$$
\frac{P_{s t}\left(\Psi_{k}\right)}{\Delta V\left(\Psi_{k}\right)}=\frac{k_{2} P_{s t}\left(\Psi_{k_{0}}\right)}{k_{2} \Delta V\left(\Psi_{k_{0}}\right)}=\frac{P_{s t}\left(\Psi_{k_{0}}\right)}{\Delta V\left(\Psi_{k_{0}}\right)}
$$

This implies that the ratio of the flicker level $P_{s t}$ to the voltage difference $\Delta V$ is independent of the impedance angle $\Psi_{k}$. This ratio depends only on the spectrum of the voltage fluctuations, and so on the spectrum of both the sea-state filtered by the response of the wave energy device considered. Equation (20) implies also that:

$$
P_{s t}\left(\Psi_{k}\right)=P_{s t}\left(\Psi_{k_{0}}\right) \frac{\Delta V\left(\Psi_{k}\right)}{\Delta V\left(\Psi_{k_{0}}\right)} \forall \Psi_{k}
$$

Hence, the flicker level $P_{s t}\left(\Psi_{k}\right)$ corresponding to a given impedance angle $\Psi_{k}$ is equal to the product of the flicker level $P_{s t}\left(\Psi_{k_{0}}\right)$ corresponding to impedance angle $\Psi_{k_{0}}$ by the ratio of the voltage differences $\Delta V\left(\Psi_{k}\right)$ and $\Delta V\left(\Psi_{k_{0}}\right)$. This formula has been used in the paper to estimate $P_{s t}\left(\Psi_{k}\right)$ from known $P_{s t}\left(\Psi_{k_{0}}\right)$ and $\Delta V\left(\Psi_{k_{0}}\right)$ by means of few simple load flow calculations.

\section{B. Equivalent sinusoidally-modulated voltage profile}

Any voltage profile inducing a flicker level $P_{s t}$ has an infinite number of equivalent sinusoidally-modulated voltage profiles $v_{e q}(t)$ which generate the same flicker level. They can be described mathematically as:

$$
v_{e q}(t)=\left(V_{a, e q}+\frac{A}{2} \sin \left(\omega_{e q} t\right)\right) \sin \left(\omega_{g} t\right)
$$

If the term $A$ of this sinusoidally-modulated voltage profile is equal to the voltage difference $\Delta V$ of the voltage profile $v(t)$, in virtue of (20), this voltage profile $v_{e q}(t)$ can be considered as equivalent to the voltage profile $v(t)$. This statement is valid for any impedance angle $\Psi_{k}$. This means that the equivalent voltage profile $v_{e q}(t)$ of any voltage profile $v(t)$ based on a reference voltage profile $v_{0}(t)$ presents the same radian frequency $\omega_{e q}$ regardless of the impedance angle $\Psi_{k}$. Only its amplitude $A$ has to be modified in order to match the voltage difference $\Delta V$ of $v(t)$ which depends on the impedance angle $\Psi_{k}$. Hence, equivalent voltage profiles can be used as an alternative for estimating the flicker level generated by more realistic voltage profiles $v(t)$ but which are far more difficult to obtain due to the reasons mentioned earlier.

The period $T_{e q}=2 \pi / \omega_{e q}$ of the sinusoidally-modulated voltage profile cannot be determined analytically. However, it must be noted that there is a high level of coupling between the input wave power and the electrical power output in the case where storage is unavailable both in the device energy conversion chain and in the farm. Hence, the period $T_{e q}$ can reasonably be assumed to be in the order of magnitude of the mean period $T_{e}$ of the sea-state considered, usually referred to as the energy period. It is calculated as [22]:

$$
T_{e}=\frac{m_{-1}}{m_{0}} \text { with } m_{k}=\int f^{k} S(f) \mathrm{d} f
$$

where $m_{k}$ is the $k^{\text {th }}$ moment of the considered sea-state spectrum $S(f)$ and $f$ is the frequency of each sinusoidal wave. In the case where storage is available either from the wave energy devices or from the wave energy farm, the level of coupling between the input wave power and the electrical power output is dramatically reduced. Hence, it can be expected that the period $T_{e q}$ is not strongly linked, in this case, to the sea-state spectrum. So, it is necessary to model the influence of storage on the flicker level. It is proposed to use a first-order low-pass filter whose time constant $\tau$ will be referred to as the "storage time constant" in the rest of the paper. The empirical validation of this approach is presented in Section IV-B.

\section{Modeling AND NUMERICAL SIMUlations SET-UP}

\section{A. Introduction}

Experimental data in the form of electrical output power profiles were provided as an outcome of the European FP7 project CORES, standing for "Components for Ocean Renewable Energy Systems" [23]. The project itself was based on a quarter-scale floating oscillating water column (OWC) which was deployed at sea off the west coast of Ireland during three months between March and May 2011. The device was connected to a small on-board island grid independent from the national electrical network.

Four different, 10-minute long electricity generation periods referred to as A, B, C, and D were considered for this study. Their corresponding sea-state characteristics are shown in Table I. The significant wave height $H_{s}$ is defined as the mean wave height (trough to crest) of the highest third of the waves [22]. The energy period $T_{e}$ was already defined in (23). During these production periods, the OWC was operated either in fixed speed mode (for production periods A and B) or in variable speed mode (for production periods $C$ and $D$ ). In other words, production periods $\mathrm{A}$ and $\mathrm{B}$ correspond to a case where little to no storage is available. On the contrary, production periods $\mathrm{C}$ and $\mathrm{D}$ correspond to a case where a significant amount of storage (in the form of inertial storage) is available and whose corresponding inertia time constant is approximately equal to $1.7 \mathrm{~s}$. This represents the inertia of the air turbine which is shown in Table II and which constitutes 
TABLE I

CHARACTERISTICS OF PRODUCTION PERIODS A, B, C AND D

\begin{tabular}{|c|c|c|c|}
\hline Production period & $H_{s}(\mathrm{~m})$ & $T_{e}(\mathrm{~s})$ & Speed control mode \\
\hline A & 5.0 & 10.9 & fixed \\
B & 2.1 & 7.3 & fixed \\
C & 4.5 & 8.8 & variable \\
D & 4.5 & 10.1 & variable \\
\hline
\end{tabular}

most of the device inertia. The power system simulations were performed using DIgSILENT numerical tool "PowerFactory" for different impedance angles $\Psi_{k}\left(30^{\circ}, 50^{\circ}, 70^{\circ}\right.$ and $\left.85^{\circ}\right)$ and for different farm rated power values equal to $5 \mathrm{MW}, 10 \mathrm{MW}$, $15 \mathrm{MW}, 20 \mathrm{MW}, 30 \mathrm{MW}, 40 \mathrm{MW}$ and $50 \mathrm{MW}$. Also, simulations have been performed for 4 production periods. In total, this represents 112 cases. These dynamic load flow simulations are based on the CORES experimental data and solved by means of the Newton-Raphson algorithm. The voltage time series obtained from PowerFactory were then processed by a flickermeter compliant with IEC standard 61000-4-15 whose design is detailed in a previous work [9]. This previous work describes also the numerical model of the wave energy farm as well as of the local electrical network which based on the Irish wave test site called AMETS [24].

\section{B. Modeling of the influence of storage on flicker}

In variable speed mode, unlike in fixed speed mode, a significant amount of inertial storage is available to smooth the output power of the wave energy devices, thus reducing the level of flicker induced by the farm. As mentioned in Section III-B, this influence of storage on the output power is proposed to be modeled by means of a first-order low-pass filter, whose time constant $\tau$ is approximately equal to the inertia time constant of the system and is thus referred to as the "storage time constant".

The validity of this approach was confirmed by comparing the flicker level induced by the wave energy farm for two production periods of similar sea-state characteristics. During the first production period, the wave energy devices were operated in variable speed mode whereas in the second production period, the devices were operated in fixed speed mode and their output power was filtered by a first-order low-pass filter. The time constant $\tau$ of this filter (i.e. the storage time constant) was chosen equal to $1.7 \mathrm{~s}$ which corresponds to the inertia time constant of the air impulse turbine (representing most of the device inertia). The flicker levels obtained for these different production periods are shown in Fig. 4 and labeled as "Storage: experimental" and "Storage: simulated" as a function of the impedance angle $\Psi_{k}$. It appears clearly that the flicker levels obtained through the simulations ("Storage: simulated") are relatively close to the flicker levels obtained based on the experimental power profiles ("Storage: experimental") as their difference does not exceed a negligible flicker level of 0.05 . Hence, the approach consisting in using a first-order low passfilter for modeling the influence of storage on the flicker level induced by a wave energy farm can be considered as valid. It was then used to generate additional fictive production periods corresponding to a variable amount of storage, i.e. to different

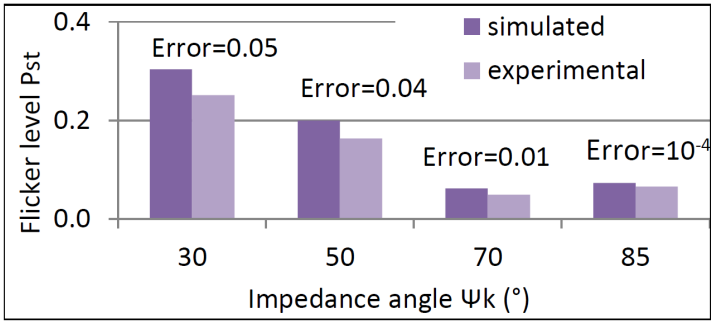

Fig. 4. Flicker level induced by a wave energy farm as obtained through the simulated approach using a first-order low-pass filter ("Storage: simulated") and through the experimental approach ("Storage: experimental")

TABLE II

STORAGE TIME CONSTANTS $\tau$ OF DIFFERENT STORAGE MEANS USED IN THE WAVE ENERGY INDUSTRY, AS FOUND IN THE LITERATURE

\begin{tabular}{|c|c|c|}
\hline Storage means & $\begin{array}{c}\text { Inertia time } \\
\text { constant (s) }\end{array}$ & Description \\
\hline Impulse turbine & 1.7 & $\begin{array}{c}\text { Calculated from full-scale } \\
\text { design concept [14] }\end{array}$ \\
\hline Hydraulic accumulator & 2 & $\begin{array}{c}\text { Volume: 50 L, } \\
\text { pressure: 10-35 MPa [14] }\end{array}$ \\
\hline Wells turbine & 3.4 & $\begin{array}{c}\text { Calculated from full-scale } \\
\text { design concept [14] }\end{array}$ \\
\hline Supercapacitor bank & $5.4-10.8$ & $\begin{array}{c}\text { Combination of } \\
13 \text { modules [8] }\end{array}$ \\
\hline Wells Turbine + flywheel & 27 & LIMPET assembly [14] \\
\hline Reservoir & 44 & $7000 \mathrm{~m}^{3}$ reservoir [14] \\
\hline
\end{tabular}

values of the storage time constant $\tau$ ranging from $0 \mathrm{~s}$ (i.e. no storage) to $5 \mathrm{~s}$ based on a single production period for which the devices are operated in fixed speed mode. It must be noted that modeling the influence of storage by means of a first-order low-pass filter is not specific to inertial storage but is valid for any type of storage (electric, hydraulic, etc.) [14]. The storage time constant corresponding to different storage means, as found in the literature is shown in Table II.

\section{RESULTS}

\section{A. Relation of linearity between $P_{\text {st }}$ and $\Delta V$}

Fig. 5 shows the flicker levels as a function of their corresponding voltage difference $\Delta V$, as well as linear approximations calculated for each production period (labeled "Lin. app."). These linear approximations present a high level of correlation $R^{2}$ with the simulation results as it is greater than or equal to 0.97 in each case. This confirms the hypothesis summarized by (21) that the flicker level follows an approximately linear relation as a function of the maximum voltage difference $\Delta V$. It must be noted that this relation of linearity had already been highlighted in [2], [15] and [16] in the case of rectangular voltage profiles only.

\section{B. Equivalent sinusoidally-modulated voltage profile}

As detailed in the theoretical analysis (Section III-B), there exists a sinusoidally-modulated voltage profile which can be considered as equivalent, in terms of flicker generation, to a given voltage profile for any impedance angle $\Psi_{k}$. Although the period $T_{e q}$ of this equivalent voltage profile could not be determined analytically, it was expected that this period would be of the order of magnitude of the energy period $T_{e}$ of the

\footnotetext{
${ }^{1}$ considered in this study
} 


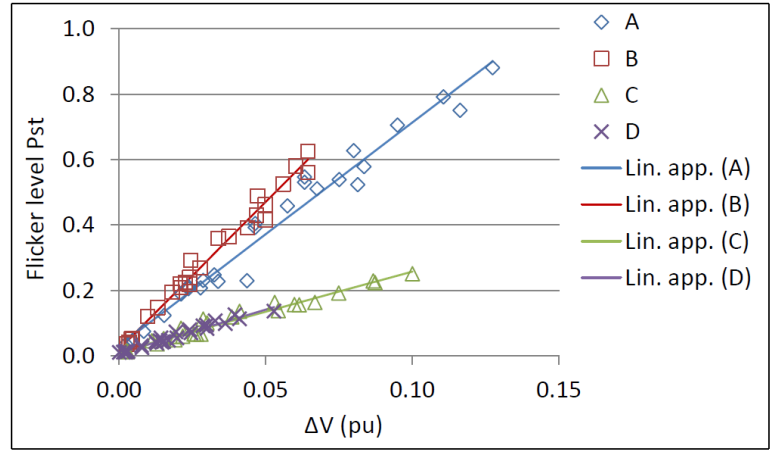

Fig. 5. Flicker level versus $\Delta V$ for the four production periods considered

sea-state in the case of production periods $\mathrm{A}$ and $\mathrm{B}$, due to the high level of coupling between the input wave power and the output electrical power. It was also expected that this may not be the case for production periods $\mathrm{C}$ and $\mathrm{D}$, due to the lower level of coupling between the input wave power and the output electrical power in this case. The value of period $T_{e q}$ was determined iteratively and the results obtained are shown in Table III. The procedure consisted in calculating expected flicker levels $P_{\text {st,exp }}$ for different values of the voltage difference $\Delta V$. The values for $\Delta V$ were selected to range between $0.01 \mathrm{pu}$ and $0.1 \mathrm{pu}$, as this range includes all the voltage difference values observed in the load flow simulations performed for this study. Following this, the corresponding expected flicker levels $P_{s t, e x p}$ were calculated for each seastate such as:

$$
P_{s t, e x p}=a\left(T_{e}\right) \Delta V
$$

where $a\left(T_{e}\right)$ depends on the energy period $T_{e}$ only and is derived from the linear approximations done for each sea-state and shown in Fig. 5. Then, the flicker levels corresponding to sinusoidally-modulated voltage profiles of different periods $T$ were computed by means of the flickermeter compliant with IEC standard 61000-4-15. For each sea-state, the period $T$ of the sinusoidal voltage profiles was optimized so that for a given voltage difference $\Delta V$, the error between the corresponding flicker level and the expected flicker level $P_{s t, \exp }$ was sufficiently small (in this study, an error $\left|P_{s t}-P_{s t, e x p}\right|$ of less than 0.01 was arbitrarily considered as acceptable). The period $T$ corresponding to this level of error is the equivalent period $T_{e q}$. Finally, as expected from (21), the equivalent period $T_{e q}$ was the same for each voltage difference value $\Delta V$. The results of this study confirmed the existence of an equivalent sinusoidally-modulated voltage profile. For the sake of illustration, a voltage profile corresponding to production period $\mathrm{A}$ and its equivalent sinusoidally-modulated voltage profile are shown in Fig. 6. The assumptions made on the relation between period $T_{e q}$ of the sinusoidally-modulated voltage profile and the energy period $T_{e}$ of the sea-state considered were also confirmed. Period $T_{e q}$ is relatively close to the energy period $T_{e}$ for production periods $\mathrm{A}$ and $\mathrm{B}$ : their ratio $T_{e q} / T_{e}$ is equal to $95.4 \%$ and $106.8 \%$ respectively. For these production periods, it was expected that $T_{e q} \leq 2 T_{e}$, as a wave device produces power twice per wave cycle in an ideal case. However, under the conditions considered in this
TABLE III

EQUIVALENT PERIOD $T_{e q}$ AND SEA-STATE ENERGY PERIOD $T_{e}$

\begin{tabular}{|c|c|c|c|}
\hline Production period & $T_{e q}(\mathrm{~s})$ & $T_{e q} / T_{e}(\%)$ & Speed control mode \\
\hline A & 10.4 & 95.4 & fixed \\
B & 7.8 & 106.8 & fixed \\
C & 29.9 & 339.7 & variable \\
D & 26.8 & 265.3 & variable \\
\hline
\end{tabular}

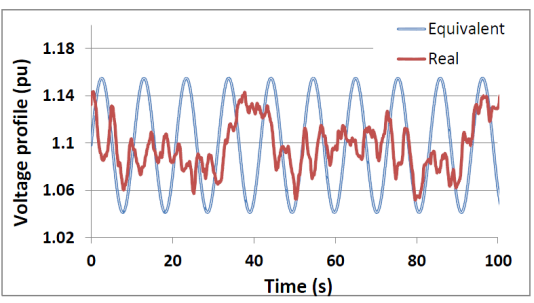

Fig. 6. Zoom on a real voltage profile and its sinusoidally-modulated equivalent in terms of flicker (production period A)

study, a number of factors reduces the coefficient of linearity between $T_{e q}$ and $T_{e}$ to lower values. In other words, only a fraction of the available wave energy is transformed into electrical energy and then into flicker. This is due among others to limitations in the wave energy capture, in losses both in the energy conversion chain and in the electrical power between the wave farm and the point of common coupling where flicker is measured. As regards production period $\mathrm{C}$ and $\mathrm{D}$, the ratio between $T_{e q}$ and $T_{e}$ does not follow this rule, as expected. In these cases, the ratio is approximately equal to $339.7 \%$ and $265.3 \%$ of the energy period $T_{e}$ respectively .

It is also interesting to note that the value of either the energy period $T_{e}$ or of the peak period $T_{p}$ could be used for defining the sinusoidal voltage profile. A previous work [17] showed indeed that no significant difference in terms of flicker level estimation appeared when one parameter was used instead of the other. Hence, it was arbitrarily decided to use the energy period $T_{e}$ in this paper.

\section{Estimation of flicker level with storage}

The relation between the period $T_{e q}$ and the energy period $T_{e}$ is not trivial in the case where a significant amount of storage is included in the device energy conversion chain, as it is the case for production periods $\mathrm{C}$ and $\mathrm{D}$, or in the wave farm. As mentioned in the previous section, this can be reasonably explained by the fact that the level of coupling between the input wave power and the electrical output power is dramatically reduced in this case. The results regarding the ratio of the flicker level $P_{s t, s}$ (corresponding to the case where storage is available) to the flicker level $P_{s t, n s}$ (corresponding to the opposite case) as a function of the storage time constant $\tau$ are shown in Fig. 7. This ratio follows a decreasing trend, as expected. It can also be noted that using storage means whose inertia time constant is equal to few seconds only can reduce flicker sufficiently for power quality requirement to be met. The ratio $P_{s t, s} / P_{s t, s}$ can be approximated with a high level of correlation by a power law but also by an exponential law. In order to extract a general law applicable to all wave energy devices, the relation between the flicker level ratio $P_{s t, s} / P_{s t, n s}$ and the storage time constant $\tau$ is currently being investigated 


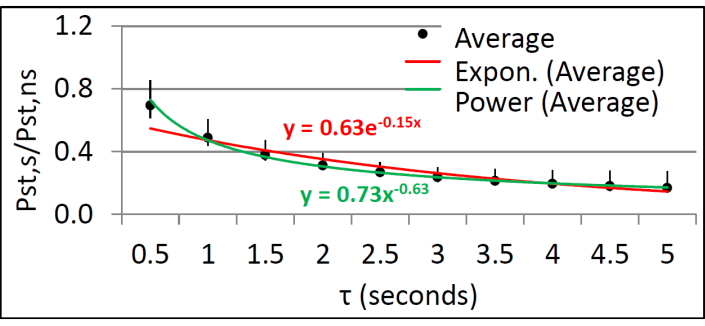

Fig. 7. Flicker level as a function of the storage time constant $\tau$ (s), as well as two approximations (power and exponential)

based on power profile data corresponding to different device technologies.

\section{CONCLUSiON}

The flicker level induced by a wave energy farm is estimated by means of a sinusoidally-modulated voltage profile. The period $T_{e q}$ of this sinusoidally-modulated voltage profile is approximately equal to the energy period $T_{e}$ of the sea-state considered. Its amplitude must be equal to $\Delta V / 2$ where $\Delta V$ is the voltage difference of the real voltage profile. This parameter can be determined by means of load flow analyses knowing the extrema of the complex power generated by the farm and the characteristics of the farm electrical network. In addition, in the case where a significant amount of storage is available, estimating the flicker level $P_{s t, s}$ is performed based on the flicker level corresponding to the fictive case where little to no storage is available $\left(P_{s t, n s}\right)$, and then using the relation linking the flicker level ratio $P_{s t, s} / P_{s t, n s}$ to the storage time constant $\tau$. Hence, the sea-state energy period $T_{e}$, the complex maximum and minimum power levels output by the farm, and potentially the storage time constant $\tau$ are sufficient to evaluate the flicker level generated by a wave energy farm.

\section{ACKNOWLEDGMENT}

The work of Anne Blavette and Dara O'Sullivan was supported through the Charles Parsons Initiative by Science Foundation Ireland which is gratefully acknowledged.

\section{REFERENCES}

[1] IEC standard 61400-21, Measurement and assessment of power quality characteristics of grid connected wind turbines, ed2.0, 2008.

[2] IEC standard 61000-4-15, Flickermeter - Functional and design specifications, ed 2.0, 2010.

[3] C. Rong et al., "Flickermeter used for different types of lamps", in Proc. $9^{\text {th }}$ Int. Conf. on Electrical Power Quality and Utilisation, Barcelona, Spain, 2007.

[4] B. Heffernan et al., "LED replacement for fluorescent tube lighting", in Proc. Australasian Univ. Power Eng. Conf., Perth, Australia, 2007.

[5] D. Gallo et al., "On the use of the flickermeter to limit low-frequency interharmonic voltages", IEEE Transactions on Power Delivery, vol. 23, pp. $1720-1727,2008$

[6] T. Keppler et al., "Theoretical assessment of light flicker caused by suband interharmonic frequencies", IEEE Transactions on Power Delivery, vol. 18, pp. 329-333, 2003.

[7] A. Hernandez et al., "A new frequency domain approach for flicker evaluation of arc furnaces", IEEE Transactions on Power Delivery, vol. 18, pp. 631-638, 2003.

[8] J. Aubry, "Optimisation du dimensionnement d'une chaîne de conversion électrique directe incluant un système de lissage de production par supercondensateurs. Application au houlogénérateur SEAREV", PhD thesis, Ecole Nationale Supérieure de Cachan, France, 2011.
[9] A. Blavette et al., "Impact of a medium-size wave farm on grids of different strength levels", IEEE Trans. on Power Systems, vol. 29, issue 2, pp. 917-923, March 2014.

[10] F. Sharkey et al., "Resource-induced voltage flicker for wave energy converters - assessment tools", IET Renewable Power Generation, vol. 7 , issue 6, pp. 623-630, May 2013.

[11] Å. Larsson, "The power quality of wind turbines", PhD thesis, Chalmers University of Technology, Sweden, 2000.

[12] T. Kovaltchouk et al., "Wave farm flicker severity: comparative analysis and solutions", Renewable Energy, submitted.

[13] Y. Coughlan et al., Wind turbine modelling for power system stability analysis-A system operator perspective, IEEE Trans. on Power Systems, vol. 22, issue 3, pp. 929-936, Aug. 2007.

[14] D. O'Sullivan et al., Dynamic characteristics of wave and tidal energy converters \& a recommended structure for development of a generic model for grid connection, technical report (OES-IA Annex III), 2010.

[15] J.J. Gutierrez, J. Ruiz and S. Ruiz de Gauna, Linearity of the IEC flickermeter regarding amplitude variations of rectangular fluctuations, IEEE Trans. on Power Delivery, Power Eng. Letters, Vol. 22, No 1, 2007.

[16] J. Majchrzak and G. Wiczynski, Basic characteristics of the IEC fickermeter processing, Modelling and Simulation Eng, 2012.

[17] A. Blavette, et al., "Estimation method for evaluating the wave-induced flicker level emitted by a tidal energy farm", in Proceedings of the $11^{\text {th }}$ European Wave and Tidal Energy Conference, Nantes, France.

[18] M. Santos, et al., "Grid integration of wave energy farms: Basque Country study", in Proc. $3^{\text {rd }}$ Int. Conf. Ocean Energy, Spain, 2010.

[19] A. Nambiar et al., "Effects of array configuration, network impacts and mitigation of arrays of wave energy converters connected to weak, rural electricity networks", in Proc. $3^{\text {rd }}$ Int. Conf. Ocean Energy, Spain, 2010.

[20] A. Blavette et al., "Wave energy grid integration in Ireland - A case study", in Proc. $3^{\text {rd }}$ Int. Conf. Ocean Energy, Bilbao, Spain, 2010.

[21] J. Cruz, Ocean wave energy, Springer, 2008.

[22] L. Holthuisjen, Waves in oceanic and coastal waters, Cambridge University Press, 2007.

[23] R. Alcorn, et al., "FP7 EU funded CORES wave energy project: a coordinators perspective on the Galway Bay sea trials", Underwater Technology, vol. 32, no. 1, pp. 51-59, March 2014.

[24] Website of the SEAI, http://www.seai.ie/Renewables/Ocean_Energy/ Belmullet_Wave_Energy_Test_Site/, Accessed September, 302014.

Anne Blavette received her $\mathrm{PhD}$ in Electrical Engineering from University College Cork, Ireland in 2013. She is currently working as a Senior Research Fellow at SATIE (CNRS unit 8029), Ecole Normale Supérieure, Rennes, France.

Dara O'Sullivan received his $\mathrm{PhD}$ from University College Cork, Ireland in 2001. He worked as a Senior Research Fellow at the Hydraulics \& Maritime Research Centre from 2007 to 2012. He is now with Analog Devices, Cork.

Ray Alcorn received his PhD from Queen's University Belfast, UK in 2000. $\mathrm{He}$ joined the Hydraulics \& Maritime Research Centre (now included in MaREI) in 2006 as a Research Manager where he was appointed Executive Director in 2011 .

Michael G. Egan received his $\mathrm{PhD}$ from the National University of Ireland, Dublin, Ireland, in 1985. Now retired, he was the director of the Power Electronics Research Laboratory, Ireland and a lecturer at University College Cork, Ireland.

Tony Lewis received his $\mathrm{PhD}$ from the University of Wales, Bangor in 1983. He is the founder of the Hydraulics \& Maritime Research Centre, now included in MaREI, Ireland. 